\title{
The Use of Tin Plague in The Analysis of Pure Tin
}

\author{
Styrkas $\mathrm{AD}^{*}$ \\ Institute of Solid-state Physics RA Sciences, Chernogolovka, Russia
}

*Corresponding author: Styrkas AD, Institute of Solid-state Physics RA Sciences, Chernogolovka, Russia

\begin{abstract}
Study focuses on the basis of knowledge the mechanism of the process $\beta \mathrm{Sn} \rightarrow \alpha \mathrm{Sn}$ for use it to analysis of important material for science and technology. The possibility of ultra-high purity Sn to analyse by measuring the rate (V) of the allotropic changing $(\mathrm{V} \beta \mathrm{Sn} \rightarrow \alpha \mathrm{Sn}$ ) is investigated. Metals of such high purity are inaccessible to chemical method, so analyzed by method of a residual resistance at temperature (T) of liquid He, inaccessible to most enterprises. The method gives an estimate of the total content of impurities. For $\mathrm{Sn}$ with low $\mathrm{T}$ of $\beta \mathrm{Sn} \rightarrow \alpha \mathrm{Sn}$ ) due to the simplicity of the measuring purity by the $\mathrm{V}$ ( $\beta \mathrm{Sn} \rightarrow \alpha \mathrm{Sn}$ ) is tempting. In high purity Sn with a low content of impurities, this method seems more accessible and convenient than others and probably possible. This paper proposes the affordable and simple method of analysis, high sensitivity, accuracy and reproducibility of the results. not inferior to the complex method of measuring the residual resistance.
\end{abstract}

Keyword: Residual Resistance; Phase Transition Rate; Impurities

\section{Introduction}

The World made 7 metals, according to the 7 planets. (Navoi). In the table of ranks of the ancient $\mathrm{Sn}$ is pair to Jupiter, the largest planet. And now Sn with the honorary № 50 in the center of the Periodic Table of Mendeleev. Sn is the oldest to man known metal. Aristotle knew about the Sn plague, but didn't know that it was a consequence of the allotropic transformation of Sn white to gray, $\beta \rightarrow \alpha$. The nebulous mysteries of Sn plague infection accumulated interests many centuries to this phenomenon. A main Interest in $\beta S n \rightarrow \alpha S n$ appeared after the evidence [1,2] Goryunova semiconductor nature of $\alpha \mathrm{Sn}$ with covalent bond by changing the metal bond to covalent, the electronic structure $s^{2} p^{2} \beta S n$ to $s^{3}$, tetragonal structure with $\mathrm{KN}=6$ to a cubic structure with $\mathrm{KN}=4$ with bonds to the vertices of tetrahedrons of $\alpha \mathrm{Sn}$. These principles creating of semiconductor compounds of needs properties. To turn into metastable $\alpha \mathrm{Sn}$ except T below $12.4^{\circ} \mathrm{C}$, is a necessary [2] seed with the parameters of the bond and structures related $\alpha \mathrm{Sn}$ and its contact with tin. The nearest neighbors of Sn give a compounds InSb and CdTe, There, pairs of atoms give in sum of total electrons the same as 2 atoms of Sn and parameters of structures [1] almost the same of $\alpha \mathrm{Sn}$. InSb, CdTe, $\alpha \mathrm{Sn}$ the better seed of $\mathrm{Sn} \rightarrow \alpha \mathrm{Sn}$, but in contrast to metastable $\alpha \mathrm{Sn}$ powder, InSb, CdTe are strong solid crystals. The infection is caused by atomic contact with a seed. Tin always covered by protective film of $\mathrm{SnO}_{2}$ which don't allow contact. If the seed is placed on the surface of $\mathrm{Sn}$, there is Infection!? And from inert substances that had contact previously with the seed although it now removed [3]. Solid crystals recognized the past! Infection at a distance is possible too! [4]. It was quite misunderstood: what gives an information from the seed? Necessary presence of the air, atmosphere. There is $I_{c}$ agent, [5-7] Inthe vacuum, dry vessel, or after treatment of the inert substance with any solvent of water, so there is no infection, $I_{c}$ is a carrier from the seed. Metastable structure $I_{c}$ in the size of nanoparticles can growing epitaxially on the related structure, penetrate through the microdefects of the protective $\mathrm{SnO}_{2}$. So, it is clear that infection under water which absorbed the $I_{c}$ nanoparticles is impossible. This opinion turned out to be wrong. With a very small probability for a time more a year under moving water, infection occurs, and this valuable phenomenon gives ways to many practical tasks and understanding of life processes [7]. The source of infection has been found. and yet another unexpected source of infection was found. This is property for practical aim. Tin remember about stay in the $\alpha \mathrm{Sn}$ phase. There is a $\beta \mathrm{Sn} \rightarrow \alpha \mathrm{Sn}$ transition and back $\alpha \mathrm{Sn} \rightarrow \beta \mathrm{Sn}$, due to a change of .$>/<d$ by $26.6 \%$.volume effect. At each $\beta \rightarrow \alpha$ move decreased $d$ and at $\alpha \rightarrow \beta d$ increased. So without external tools $S n$ gives pure powder of any size particles $[8,9]$. 
Knowing the $\mathrm{I}_{\mathrm{c}}$ as seed allows to use for solving a row of other practical problems [5-7]with use of the terrible plague by a simple way $[10,11]$ in forms convenient for creating $p / n$ shifts , simple effective purification of Sn without melting in solid phase [12]. Method of zone melting [13] to purification is determined by the difference in the $\mathrm{K}$, ratio of the solubility of impurities at the phase boundary. At melting metal doesn't change type of the bond on the border of solid/liquid, soK is near to 1 , the difference is knowingly less than at of the metal /semiconductor boundary with the great differences in the nature of their chemical bonds, CN (coordination number), structures. The cleaning efficiency at the border metal / semiconductor, $\mathrm{K}$ far from 1 . And so was a reason that zone melting became widely used when there was a need in semiconductors of high purity. A knowledge of the mechanism of the solid-phase process of $\beta S n \rightarrow \alpha \mathrm{Sn}$ [7] land to opinion of possibility to apply it in the analysis of the height purity of $\mathrm{Sn}$.

\section{Theoretical View on The Possibility of Analyzing by V Bsn $\rightarrow$ Asn}

Analysis of high-purity materials is labor-intensive and often impossible if the sensitivity of classical methods is insufficient [14]. There is a method for measuring the $\gamma_{4.2 \mathrm{~K}}$, i.e. the ratio $\mathrm{R} 300 \mathrm{~K} / \mathrm{R}$ $4.2 \mathrm{~K}$, method of residual resistance, which gives an estimate of the amount of impurities in metals [15] of high purity. The residual resistance of Sn at $4.2 \mathrm{~K}$ before the transition to the superconducting state depends on its purity and perfection of structure. The $\mathrm{R}$ at $\mathrm{T}$ of room is almost constant, and the $\gamma_{4.2 \mathrm{~K}}$, i.e. the ratio $\mathrm{R} 300 \mathrm{~K} / \mathrm{R} 4.2$ $\mathrm{K}$, is residual $\mathrm{R}$ characterizes the purity of $\mathrm{Sn}$. The purer the metal and more perfect its structure, the lower the $\mathrm{R}$ at $4.2 \mathrm{~K}$ and the higher the value $\gamma_{4.2 K}$, which serves as a measure of the total content of impurities in metals. But measuring equipment is difficult, and liquid He is rarely available to the most of organizations. Studies of allotropic transformation of Sn [5-7] showed a connection between the purity by $\gamma_{4.2 \mathrm{~K}^{\prime}}$ and the rate $\mathrm{V}$ of its phase transformation into $\alpha \mathrm{Sn}$. But also, it seemed unrealistic to use it for analyses after bright experiments [16] showed the impurities in Sn are accelerating, indifferent and inhibiting. Hence, the analysis of the purity of Sn by $\mathrm{V}$ $\beta S n \rightarrow \alpha S n$ is impossible at it depends on the ratio of concentrations of dissimilar impurities. But the mechanism of distinguishing the role of impurities is not clear at all. If each atom of the impurity violates the $\gamma_{4.2 \mathrm{~K}}$, of the metal, which the $\gamma_{4.2 \mathrm{~K}}$ method illustrates by analyzing any other metals, why the impurities of different metals differ in their effect on the $\mathrm{V} \beta \mathrm{Sn} \rightarrow \alpha \mathrm{Sn}$ transition. This became clear when we knew the mechanism of infection with the "tin plague" [4]. In [16] was studied Sn not of high purity, there are no errors in experiments. The chaotic nature of the dependences of $\mathrm{V}$ on purity is clearly shown [5,7] at studying the influence of impurities on $\mathrm{V}$ of $\beta S n \rightarrow \alpha S n$. The fact is that the commonly zone melting is powerless to clean from $\mathrm{Sb}$ because it has $\mathrm{K}=1$ in $\mathrm{Sn}$. The solubilities of $\mathrm{Sb}$ in solid and molten Sn are the same, And the Sb impurity on both sides of the phase boundary is the same and so can't to be redistributed, as other impurities with $\mathrm{K} \neq 1$.And in the ores of $\mathrm{Sn}$ impurity in the $\mathrm{Sb}$ usually dominates. At zone melting cleaning, the Sb impurity always prevails over the others. And In himself like of all metals is inhibitory too by the same reasons, but it was shown as accelerator [16] because $\mathrm{In}+\mathrm{Sb}$ gives the best seed InSb. And in the Sn of high purity, the impurity of In, like any impurity, individual. But having the knowledge about the dependence of the $\beta S n \rightarrow \alpha$ Sn process on many factors, it is necessary to observe the requirements 1-4, understood during the experiments for creating a method for analyzes [17].

\section{Experimental Part}

It is possible to create a method for analyzing the purity of $\mathrm{V} \beta \mathrm{Sn} \rightarrow \alpha \mathrm{Sn}$ similar to measurements of residual resistance, suitable for high-purity metals. Previously, it was found $[3,5,7]$ that the dependence of $\mathrm{V} \beta \mathrm{Sn} \rightarrow \alpha \mathrm{Sn}$ on $\mathrm{T}$ for any samples has a maximum. This is very easy to understand. At low T with its growth $\mathrm{V} \beta \mathrm{Sn} \rightarrow \alpha \mathrm{Sn}$ grows according to the Arrhenius equation. $\mathrm{V}$ cannot grow constantly, because as it approaches the point of the phase transition, it becomes smaller and turns to 0 . When infected, Sn crumbles into an arc-shaped powder, making difficult to measure phase shift lengths. Amorphous wires of fast quenching, single crystals of $\beta S n$ and even annealed wires with slow infection remain almost the original shape but with some bending, and break at $\mathrm{V} \beta S n \rightarrow \alpha \mathrm{Sn}$ depending on the T (Figure 1) to parts of different lengths, but almost the same at each T. Accumulation of impurities by the method of residual resistance was recorded in the fracture. It is seen that after the fracture, the sections at each $\mathrm{T}$ are close to each other. For analysis, it is necessary that the content of impurities is constant along the length, that is, choose $\mathrm{V} \beta \mathrm{Sn} \rightarrow \alpha \mathrm{Sn}$ for it, $\mathrm{V}$ of growth of $\alpha \mathrm{Sn}$ and $V$ of impurities were now equal, and $S n$ maintain the solidity too.

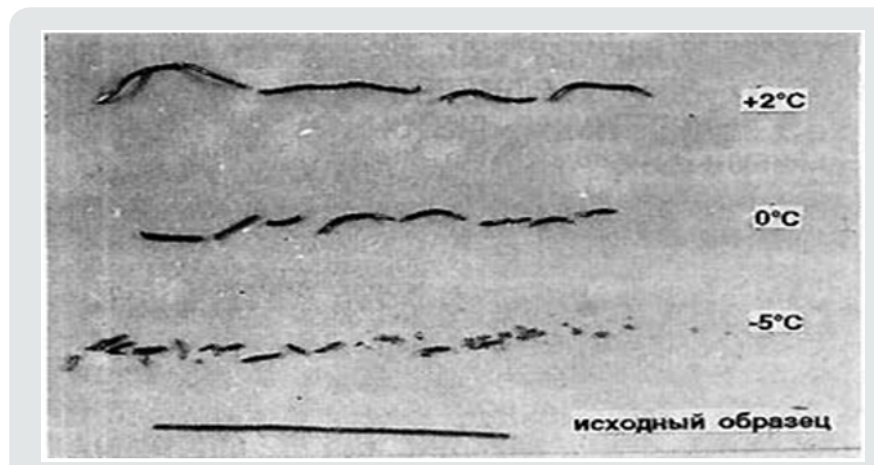

Figure 1: Fracture of Sn of different purity with the accumulation of impurities overtaking the phase boundary at its low $\mathrm{V} . \mathrm{T}=+2: 0$ and $-5^{\circ} \mathrm{C}$.

\section{Requirements}

1) Monoliths are obtained for the growth of $\alpha \operatorname{Sn}[10,11]$ in the ice shape. The study of a movement of impurities at $\beta S n \rightarrow \alpha S n$ allowed us to create a method like of zone cleaning in a solid, but for analysis it is necessary that the content of impurities is constant 
along the length, that is, choose $\mathrm{V} \beta \mathrm{Sn} \rightarrow \alpha \mathrm{Sn}$ and $\mathrm{V}$ of impurities equal and maintain the solid state.

2)Monoliths are obtained by standard preparing a Sn for analysis, so its behavior and structure depends on the previous mechanical and thermal history of Sn. Ins sample in standard quartz forms melted and cooled under standard vacuum conditions, then $\mathrm{Sn}$ melt poured into a $\mathrm{SiO}_{2}$ mold to made identical samples in the form of wire or rod with a spherical surface of one edge of it, then annealed and cooled in vacuum.

3)To create the minimum of seeds by moving of $\mathrm{H}_{2} \mathrm{O}$ near of the contact Sn of spherical surface of edge with polished or spherical surface of InSb seed.in thermostat with selected T for analysis. So, to create the minimum of seeds by moving of $\mathrm{H}_{2} \mathrm{O}$ near of contact $\mathrm{Sn}$ with InSb in thetermostat with ice nearly of chooses $\mathrm{T}$.

4)The diagram of calibration dependence of $\mathrm{V} \beta \mathrm{Sn} \rightarrow \alpha \mathrm{Sn} / \gamma_{4.2}$ should be attributed to the same strictly selected $\mathrm{T}$ for analysis.

5)The infection $V$ should be measured repeatedly for graphical correction of errors in a visual determination of the length of the infected area. At $\mathrm{T}$, chosen for a phase transition the impurity does not accumulate, and the concentration along the entire length is constant, which is important for analysis. For the integrity of the sample, it is possible to infect as in $[10,11]$. You can make many measurements $\mathrm{V} \beta \mathrm{Sn} \rightarrow \alpha \mathrm{Sn}$ on length, reducing the measurement error statistically. The sections along the path of the Sn white dark border is measured repeatedly over time. After the end of the analyze measurement with standard remelting, the $\alpha \mathrm{Sn}$ is converted to $\beta S n$, especially if the analysis result must be checked by direct measurement $\gamma_{4,2 \mathrm{~K}^{\prime}}$, which is applicable only f or metals. According to the graph for a given analysis at T V $\beta \mathrm{Sn} \rightarrow \alpha \mathrm{Sn}$ from $\gamma_{4,2 \mathrm{~K}}$ find the purity of Sn. Measures of $\mathrm{V}$ different samples gave 1.37 and $1.41 \mathrm{~mm} /$ hour, corresponded to $\gamma_{4,2 \mathrm{~K}} 47500$ and 55000 . Control analyses of them give $\gamma_{4,2 \mathrm{~K}} 46,800$ and 55,400. Errors of $1.5 \%$ and $0.8 \%$ within the measurement accuracy of $V$ and $\gamma_{4,2 K}$. And to check the reproducibility of results in 10 standard samples, an infection $\mathrm{V}$ was measured on the same day in the same thermostat. The average of a value of $V$ is $1.48 \mathrm{~mm} /$ hour. A maximum deviation $\mathrm{V}$ value of one sample was $1.46 \mathrm{~mm} /$ hour, which is $1.3 \%$, all the others gave $1.48,149,1.47$.

\section{Summary}

By using for the practical aims of "terrible tin plague" along with its application to obtain pure powders of a given dispersion, for further purification of high-purity tin, for growing profiled crystal of a unique material $\alpha \mathrm{Sn}$ even with $\mathrm{p} / \mathrm{n}$ transition, simple accessible method of purity Sn analysis was created, which seemed fundamentally impossible. The accuracy and reliability of the results of the proposed method with obvious availability, accessibly and simplicity even is not complicated and complex method of residual resistance without using of liquid helium. Here is only whether the method can be considered created until it still not published and not known to researchers, for whom, and not for corrupt officials, this work was done.

\section{Gratitude's}

The author is grateful to V. V. Ryazanov for his help in measuring $\gamma_{4.2 K}$ - residual resistance and for his constant interest in the work, advice and discussions and cooperation with N. G. Nikishina, R. A. Ohanyan, Efremov A.S, Boronina L.R., Sidelnikov M.S.

\section{References}

1. Goryunova NA (1950) On the question of $\beta \rightarrow \alpha$ the tin transition. 75: 5154.

2. Goryunova NA (1960) Chemistry of diamond-like semiconductors. Zh. All-Russian Chemical Society named after DI Mendeleev 5: 522-535.

3. Bykhovsky AI (1963) Ukr. Fiz. zhurn. 8(6): 600.

4. Kuo K, Burgers WG (1956) Proc. Konikl. Nederl. Akad. Weten, Amsterdam 59(4): 288.

5. Styrkas AD (2003) Mechanisms of the Allotropic Ttransformation of Sn. "Inorganic Materials" 2003 T 39 N8 C 944-948 Grow of Gray Tin Crystals. "Inorganic Materials 39(8): 806-810.

6. Hobbs PV (1974) Ice Physics, Clarendon Press, Oxford, 352 pp.

7. Styrkas AD (2016) "life, memory, recognition and aging" of grey tin. Journ. Materials Science and Chemical Engineering 4: 1-11.

8. Hobbs PV (1974) Ice Physics, Clarendon Press, Oxford 352 pp.

9. Styrkas AD, Oganyan RA (1992) Production and Properties of Tin Powders Powder Metallurgy 35(2): 117-119.

10. Styrkas AD, (2001) Reduction of O-Content in High-Purity Tin Powders. Powder Technology 2: 137-139.

11. Styrkas AD (2003) Grow of Gray Tin Crystals. Inorganic Materials 39(7): 683-686.

12. Styrkas AD (2005) Preparation of Shaped Gray SnCrystals. Inorganic Materials 41(3): 450-452.

13. Styrkas AD (2018) Purification of Solid tin. Instruments and Experimental Techniques 61(3): 450-452.

14. Pfann V (1969) Zone melting M Mir pp.69.

15. Spivakovsky VB (1975) Analytical chemistry Sn. M. Nauka pp.250.

16. Alexandrov BI, Dukhin VV (1972) The Influence of Impurities on the Residual Resistance of Tin. Phys.f Metals and Metallography. 34: 739749.

17. Semenchenko BK, Pokrovsky IL, Lazarev VB (1953) Influence of small impurities on polymorphic transformations in tin. SSRR 89(6): 10211024. 


\section{(c) (i) This work is licensed under Creative}

To Submit Your Article Click Here: Submit Article

DOI: $10.32474 /$ MAMS.2020.03.000153

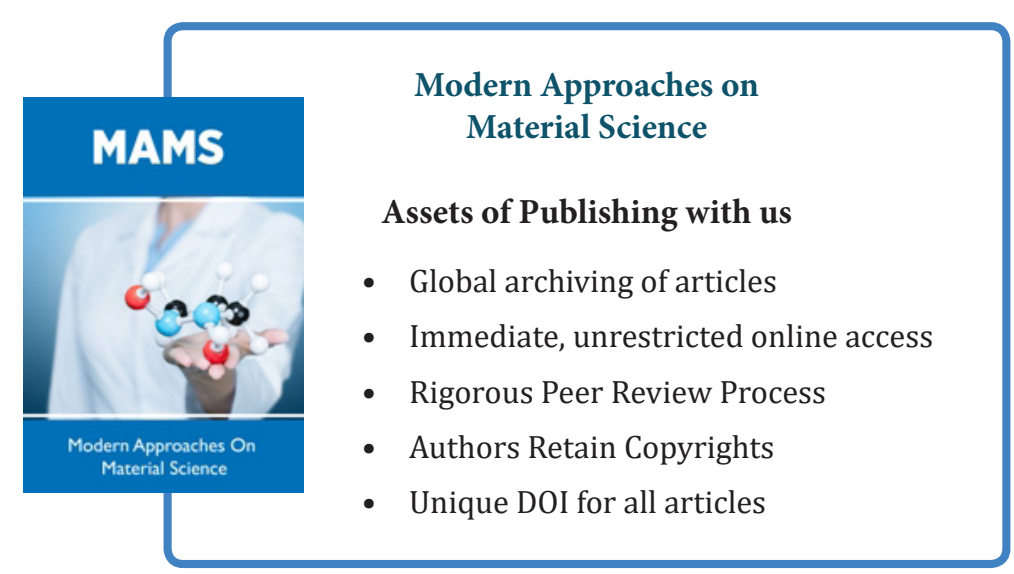

